\title{
Thoracic endovascular aortic repair improves the quality of life in young patients with traumatic aortic injury
}

\author{
kentaro kiryu ${ }^{1}$, Takayuki Kadohama ${ }^{2}$, Itaru Igarashi ${ }^{3}$, Genbu Yamaura ${ }^{3}$, Fuminobu \\ Tanaka $^{3}$, Daichi Takagi ${ }^{2}$, Yoshinori Itagaki $^{1}$, Takuya Wada ${ }^{3}$, Yuya Yamazaki ${ }^{3}$, and Hiroshi \\ Yamamoto $^{3}$ \\ ${ }^{1}$ Akita University Graduate School of Medicine School of Medicine \\ ${ }^{2}$ Akita University \\ ${ }^{3}$ Akita University Graduate School of Medicine School of Medicine Department of \\ Cardiovascular Surgery
}

May 15, 2020

\begin{abstract}
Background \& Aim: Traumatic aortic injury (TAI) is a life-threatening condition. We present cases of 7 patients with TAI limited at the isthmus. Case description: Seven patients with TAI were treated between January 2015 and December 2018; TAI was caused by motor vehicle crashes in all cases. The patient characteristics and the post-operation data were collected and analyzed. We performed thoracic endovascular aortic repair (TEVAR) for five patients during their first hospitalization using Relay Plus@ (Japan Lifeline, Japan). While patient 6 underwent TEVAR 5 years after the injury, patient 7 was recommended conservative care because she had dementia. Since most of these patients did not have a history of hypertension, they were not on antihypertensive medications after TEVAR. Conclusions: In cases of TAI, intervention by TEVAR in the acute phase improves the patient's quality of life. Additionally, TEVAR is expected to prevent TAI from enlarging to form an aortic aneurysm.
\end{abstract}

\begin{abstract}
Background \& Aim: Traumatic aortic injury (TAI) is a life-threatening condition. We present cases of 7 patients with TAI limited at the isthmus.

Case description: Seven patients with TAI were treated between January 2015 and December 2018; TAI was caused by motor vehicle crashes in all cases. The patient characteristics and the post-operation data were collected and analyzed. We performed thoracic endovascular aortic repair (TEVAR) for five patients during their first hospitalization using Relay Plus ${ }^{\circledR}$ (Japan Lifeline, Japan). While patient 6 underwent TEVAR 5 years after the injury, patient 7 was recommended conservative care because she had dementia. Since most of these patients did not have a history of hypertension, they were not on antihypertensive medications after TEVAR.
\end{abstract}

Conclusions: In cases of TAI, intervention by TEVAR in the acute phase improves the patient's quality of life. Additionally, TEVAR is expected to prevent TAI from enlarging to form an aortic aneurysm.

Keywords: Traumatic aortic injury, Thoracic endovascular aortic repair, aneurysm, dissection, trauma

\section{Introduction}

Traumatic aortic injury (TAI) is a life-threatening condition with a wide range of clinical manifestations, ranging from hemodynamic stability to fatality, and requires local aortic dissection. Most TAI patients are young and do not have a history of hypertension. However, for patients with TAI and multiple organ injuries, 
the timing of the surgical interventions is critical, unlike for patients with aneurysms due to arteriosclerosis. We present cases of 7 patients with TAI limited at the isthmus. Thoracic endovascular aortic repair (TEVAR) was performed urgently in 5 patients during their first hospitalization.

\section{Case description}

We treated 7 patients with TAI ( 5 men and 2 women; mean age $56 \pm 10$ years) between January 2015 and December 2018; TAI was caused caused by motor vehicle crashes in all cases. The patients underwent TEVAR with Relay Plus ${ }^{\circledR}$ (Japan Lifeline, Japan). The patient characteristics have been summarized in Table 1. The post-operation data for the patients are shown in Table 2.

In 5 cases, TEVAR was performed in the acute phase of the injury (Fig.1). The average time between hospitalization and surgery was 27 days. In patients 1-4, the circulation was stable, and hence computed tomography (CT) was performed first. However, patient 5 was in shock due to a superior vena cava (SVC) injury, which was treated prior to TEVAR during the same operation.

Patients 1, 2, 4, and 5 were young and did not have a history of hypertension. Therefore, after the operation, they did not require antihypertensive medications.

In patient 6 , we detected enlargement of a dissecting aortic aneurysm 5 years after the initial injury. We, therefore, could not perform Zone 3 TEVAR (Fig.2). Patient 7 had a history of dementia. Since she had no enlargement of the aorta, we just continued with follow-ups.

\section{Discussion}

TAI is the second most common cause of death after head injury. It has a wide variety of clinical manifestations ranging from hemodynamic stability to fatality. ${ }^{1}$ About $81 \%$ of the TAI cases are caused by motor vehicle crashes and have the same underlying mechanism. ${ }^{1}$ All 7 patients in our study were also injured in motor vehicle crashes.

TAI has generally been thought to be a rapidly decelerating injury. Parmley et al., in a study of 275 patients with TAI, found that $239(87 \%)$ died during the first hour. ${ }^{2}$ They also found that of the patients who arrived alive at the medical facility, $15 \%$ died from an aortic rupture within a few days.

Hershberger et al., in a study of 1126 cases of TAI, reported that in 818, the injury was in the thoracic aorta. ${ }^{3}$ Simon Sevitt reported that in all the cases of TAI studied, the injury was at the isthmus, ${ }^{4}$ which is consistent with our findings. The factors that influence the location of the TAI include ${ }^{4}$ :

1. Upward displacement of the heart and mediastinum from a cranially directed impact on the lower chest.

2. Acute deceleration of the moving body producing tension-stress on the wall of the aorta. The deceleration can be directed cranially, caudally, or horizontally.

3. A combination of direct trauma to the thorax plus effects of deceleration, as seen in many drivers and front-seat passengers after frontal collisions of their vehicles.

4. An acute rise of intra-aortic pressure following a blow on the chest or deceleration.

Azizzadeh et al. have classified TAI into 4 types, ${ }^{5}$ while Fabian et al. have classified it into 6 types. ${ }^{6}$ Azizzadeh et al. have recommended that types III and IV should be treated urgently. But in most cases, there are other injuries as well, which makes it difficult to decide the right time to operate.

The characteristics of patients with TAI are different from those with a common aortic rupture or dissection. While Hershberger et al. have reported TAI in relatively young patients with an average age of 41 years, ${ }^{3}$ Azizzadeh et al. have reported it in children. ${ }^{5}$ Compared to patients with a common aortic aneurysm, patients with TAI have a healthy aorta, no history of hypertension, and, therefore, are not on heart-related medications. The average age of the patients in our study was 56.3 years, and only 3 of them underwent any medical intervention before the onset of TAI. 
Most patients with TAI have multiple injuries. Therefore, careful examination is needed in each case to determine the right time for intervention, and patients should be monitored closely to see how they adapt to the intervention. TEVAR has recently been recommended for Class I TAI. Compared to a cardio-pulmonary bypass, TEVAR requires fewer doses of heparin.

Hershberger et al. reported mortality rates of $15-50 \%$ and $9.5 \%$ following conventional open-heart surgery and TEVAR, respectively. ${ }^{3}$ While paraplegia occurred in $9-19 \%$ of the patients who underwent open-heart surgery, it occurred in only $0.24 \%$ of those who underwent TEVAR. In a study comparing open-heart surgery and TEVAR, Ott et al. found that both the mortality rates and incidence of paraplegia were $16 \%$ and $0 \%$, respectively, in the two groups. Therefore, TEVAR is considered safe and useful, although prolonged followups are required to monitor the long-term effects of TEVAR on the patient's health. There was no incidence of mortality or paraplegia in our study.

In conservative cases, TAI has been shown to enlarge and form a dissecting aortic aneurysm, ${ }^{8,9}$ as seen in patient 5 in this study. In a study of chronic traumatic thoracic aneurysms, Finkelmeier et al. have reported that $41 \%$ of the patients had either died or had indications of expansion, 5 years after the injury. ${ }^{8}$ At 20 years, after the initial injury, there was only a $33 \%$ probability of being alive and free of signs or symptoms of aneurysm expansion. They also found that the survival rates in patients who did and did not undergo operative repair 10 years after surgery were $85 \%$ and $66 \%$, respectively. ${ }^{8}$

In this study, we have reported 7 cases of TAI localized at the isthmus, all caused by motor vehicle crashes. We performed TEVAR in the acute phase of the TAI in 5 patients, and 4 of them were in stable condition. Patient 5 showed signs of shock, and therefore, we repaired the SVC prior to performing TEVAR in the same operation. We followed-up patient 6 by CT and detected an enlarged aortic aneurysm 5 years later. If we had performed TEVAR in the acute or subacute phase, instead of in the chronic phase, we could have intervened as Zone 3 TEVAR.

Patient 7 was treated conservatively since she had dementia and was at a high risk of bleeding following the use of heparin. Patients 1, 2, 4, and 5 were young, with no history of hypertension. Therefore, they did not have to take medications for hypertension, which we believe contributed to their quality of life. However, prolonged follow-up data will have to be evaluated to determine the long-term effects of TEVAR in these patients.

Paraplegia is one of the serious complications of TEVAR. In our cases, however, TEVAR did not result in paraplegia. The collateral network concept proposed by Griepp et al. describes the presence of various collateral pathways in the spinal cord. ${ }^{10},{ }^{11}$ Therefore, covering only the aortic isthmus, as in our cases, reduces the risk of paraplegia. Furthermore, we performed a left subclavian artery bypass as a prophylactic measure against paraplegia.

Limitation of our report was the number of cases was small. So, it is necessary to continue to accumulate cases.

\section{Conclusion}

Based on the 7 cases described in this study, particularly the one involving a young patient with no history of hypertension, we believe that TEVAR performed during the acute or sub-acute phase of injury is effective. It contributes to a better quality of life by eliminating the need for antihypertensive medications and potentially prolonging the life span. Furthermore, our findings suggest that performing a simple TEVAR before the TAI progresses to a chronic stage and becomes a dissecting aortic aneurysm, is associated with fewer complications and a lower risk of paraplegia.

Conflict of interest: The authors declare no conflicts of interest directly relevant to the content of this manuscript.

\section{Author contributions Writing: KK}

Review: TK, HY 


\section{Illustration: II}

\section{Data summarize: GY, FT, DT, YI, TW, YY}

\section{References}

1. Kato N,Dake MD, Miller DC,Semba CP, Mitchell RS,Razavi MK, et al. Traumatic thoracic aortic aneurysm: treatment with endovascular stent-grafts. Radiology 1997;205:657-662.

2. Parmley LF, Mattingly TW, Manion WC, Jahnke EJ. Nonpenetrating traumatic injury of the aorta. Circulation 1958;17:1086-1101.

3. Hershberger RC,Aulivola B,Murphy M,Luchette FA. Endovascular grafts for treatment of traumatic injury to the aortic arch and great vessels. J Trauma 2009:67;660-671.

4. Sevitt S. The mechanisms of traumatic rupture of the thoracic aorta. Br J Surg 1977; 64:166-173.

5. Azizzadeh A, Ray HM, Dubose JJ, Charlton-Ouw KM, Miller CC, Coogan SM, et al. Outcomes of endovascular repair for patients with blunt traumatic aortic injury. J Trauma Acute Care Surg 2014;76:510-516.

6. Fabian TC, Richardson JD, Croce MA, Smith JS Jr, Rodman G Jr, Kearney PA, et al. Prospective study of blunt aortic injury: Multicenter Trial of the American Association for the Surgery of Trauma. J Trauma 1997;42:374-383.

7. Ott MC,Stewart TC,Lawlor DK,Gray DK,Forbes TL. Management of blunt thoracic aortic injuries: endovascular stents versus open repair. J Trauma 2004;56:565-570.

8. Finkelmeier BA,Mentzer RM Jr,Kaiser DL,Tegtmeyer CJ,Nolan SP. Chronic traumatic thoracic aneurysm. Influence of operative treatment on natural history: an analysis of reported cases, 19501980. J Thoracic Cardiovasc Surg 1982;84:257-266.

9. Bacharach JM, Garratt KN, Rooke TW. Chronic traumatic thoracic aneurysm: Report of two cases with the question of timing for surgical intervention.J Vasc Surg 1993;17:780-783.

10. Griepp RB,Griepp EB. Spinal cord perfusion and protection during descending thoracic and thoracoabdominal aortic surgery: the collateral network concept. Ann Thorac Surg 2007;83:865-869.

11. Griepp RB, Ergin MA, Galla JD, Lansman S, Khan N, Quintana C, et al.Looking for the artery of Adamkiewicz: a quest to minimize paraplegia after operations for aneurysms of the descending thoracic and thoracoabdominal aorta. J Thorac Cardiovasc Surg 1996;112:1202-1215.
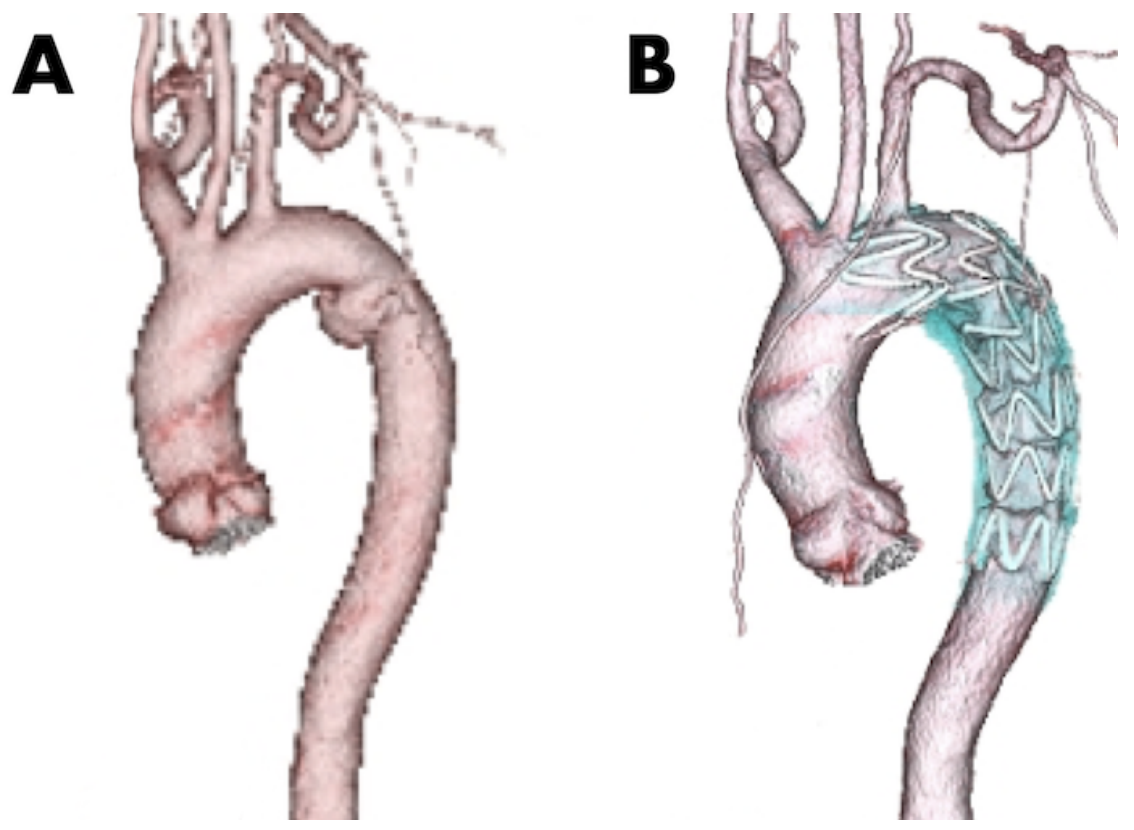

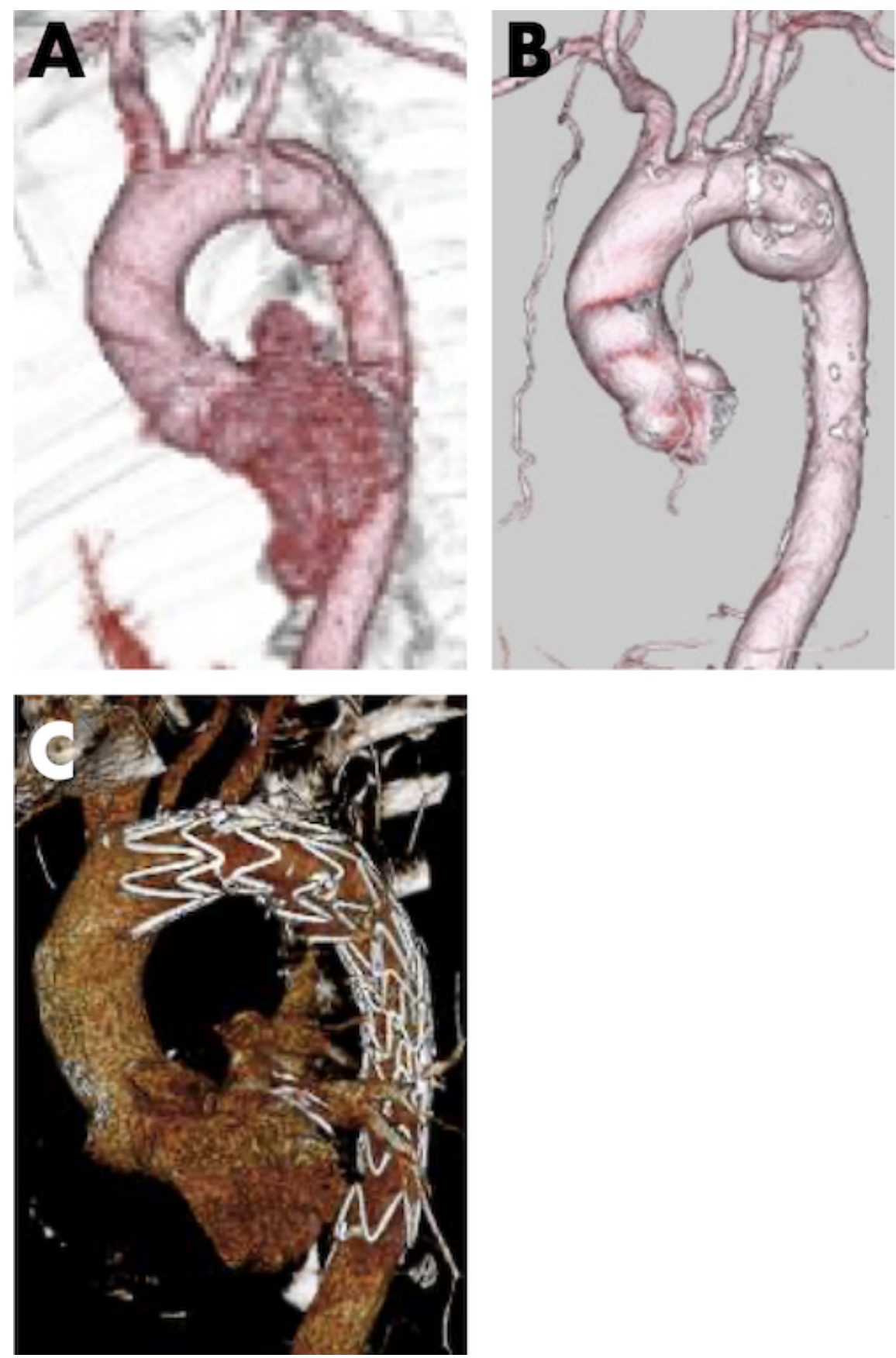

\section{Hosted file}

Table1_Patient_characteristics part5.docx available at https://authorea.com/users/316887/ articles/451318-thoracic-endovascular-aortic-repair-improves-the-quality-of-life-inyoung-patients-with-traumatic-aortic-injury

\section{Hosted file}

Table2_operative_result part5.docx available at https://authorea.com/users/316887/articles/ 451318-thoracic-endovascular-aortic-repair-improves-the-quality-of-life-in-young- 
patients-with-traumatic-aortic-injury 